\title{
ANTIBACTERIAL AND CYTOTOXIC POTENCIES OF STILBENE OLIGOMERS FROM STEM BARKS OF BAOTI (DRYOBALANOPS LANCEOLATA) GROWING IN KENDARI, INDONESIA
}

\author{
SAHIDIN I ${ }^{1 *}$, WAHYUNI $\mathbf{W}^{1}$, MUH HAJRUL MALAKA ${ }^{1}$, IMRAN I $^{2}$ \\ ${ }^{1}$ Department of Pharmacy, Faculty of Pharmacy, Universitas Halu Oleo, Kampus Baru Anduonohu, Kendari, Sulawesi Tenggara, Indonesia. \\ ${ }^{2}$ Department of Chemistry, Faculty of Mathematics and Natural Sciences, Universitas Halu Oleo, Kampus Baru Anduonohu, Kendari, \\ Sulawesi Tenggara, Indonesia. Email: sahidin02@yahoo.com \\ Received: 23 March 2017, Revised and Accepted: 27 April 2017
}

ABSTRACT

Objective: The purpose of the research is to isolate and to identify compounds from stem barks of Dryobalanops lanceolata and to evaluate their activities toward bacteria and cancer cell lines.

Methods: Isolation of the compounds worked with chromatography methods including thin-layer chromatography, vacuum liquid chromatography, and radial chromatography. All compound structures were determined based on the spectroscopic evidence including ultraviolet (UV), Infrared, and one-dimension and two-dimension nuclear magnetic resonance spectra and comparison the spectroscopy data with related data from references. The biological properties of compounds are evaluated toward bacteria Escherichia coli ATCC 35219 and Staphylococcus aureus ATCC 25923 and human breast cancer cell lines T-47D as cytotoxic potency.

Results: Five stilbene oligomers have been isolated and identified from acetone extract of $D$. lanceolata stem barks namely balanocarpol (1), $\varepsilon$-viniferin (2), $\alpha$-viniferin (3), vaticanol B (4), and hopeaphenol (5). The inhibition zone value (in mm) of $\varepsilon$-viniferin, balanocarpol, $\alpha$-viniferin, hopeaphenol, and vaticanol B toward $E$. coli and $S$. aureus is $11 \pm 0.22$ and $7 \pm 0.17,9 \pm 0.17$ and $13 \pm 0.12,8 \pm 0.20$ and $8 \pm 0.16,6 \pm 0.16$ and $8 \pm 0.11$, and $5 \pm 0.12$ and $4 \pm 0.14$, respectively. Biological activity of the compounds against breast cancer cell lines T-47D (inhibitory concentration $50 \%$ in $\mu \mathrm{M}$ ) for $\varepsilon$-viniferin, balanocarpol, $\alpha$-viniferin, hopeaphenol, and vaticanol B is $34.13 \pm 0.15,98.17 \pm 0.41,84.79 \pm 0.24,52.04 \pm 0.26$, and $119.30 \pm 0.54$, respectively.

Conclusions: $\varepsilon$-viniferin is the most active compound toward E. coli and human breast cancer cell lines T-47D. Biological activity against bacteria $S$. aureus indicated that balanocarpol is the most potential compound.

Keywords: Dipterocarpaceae, Dryobalanops lanceolata, Stilbenoids, Antibacterial, Cytotoxic.

(c) 2017 The Authors. Published by Innovare Academic Sciences Pvt Ltd. This is an open access article under the CC BY license (http://creativecommons. org/licenses/by/4. 0/) DOI: http://dx.doi.org/10.22159/ajpcr.2017.v10i8.18664

\section{INTRODUCTION}

Chemical and pharmacological study of traditional medicinal plants from Kendari,Indonesia has been conducted. The plants were Dipterocarpaceae [1-3], Jatropha [4-6], Annonaceae [7], Pongamia [8], Imperata [9], Polygonum [10,11], and Dillenia [12]. In continuing our study of both aspects, Dryobalanops genera (Dipterocarpaceae) is still interesting. Dryobalanops is a minor genus of Dipterocarpaceae and comprises 7 spesies. Conventionally, this plant used for stomachache and antioxidant [13]. The benefits showed that the chemical content and pharmacological aspects of Dryobalanops plants are very important.

Phytochemical studies of Dryobalanops, like other plants in Dipterocarpaceae such as Dipterocarpus [14] and Hopea [15], produced stilbene oligomers. Some Dryobalanops plants which have studied on chemical content are $D$. aromatica, resulted $\varepsilon$-viniferin, $\alpha$-viniferin, laevifonol, ampelopsin E, malaysianol A, flexuasol A, vaticanol B, vaticanol C, diptoindonesin A, and bergenin [16,17]. Dryobalanops beccarii gave malaysianol $\mathrm{D}$, malaysin $\mathrm{A}$, $\varepsilon$-viniferin, diptoindonesin $\mathrm{A}$, flexuasol A, vaticanol B, vaticanol C, bergenin, 4-0-galloylbergenin, scopoletin, 4-0-methylbalanophonin, methyl gallate, and gallic acid [18]. Cis- diptoindonesin B and trans-diptoindonesin B were isolated from Dryobalanops lanceolata [19]. Moreover, D. lanceolata from Malaysia yielded malaysianol B, hopeaphenol, stenophyllol, nepalensinol $B$, vaticanol $B$, vaticanol $C$, upunaphenol $D$ and flexuosol A [20]. Pharmacological study showed that $\varepsilon$-viniferin is the most active compound against HL-60 cell lines [16] and vaticanol $C$ is active toward A549 cell lines [18]. Upunaphenol D and flexuasol A displayed interisting potency toward bacteria Staphylococcus epidermidis, Staphylococcus aureus, Staphylococcus xylosus, Shigella flexneri, Salmonella typhimurium, and Escherichia coli [17]. One of the Dipterocarpaceae plants which grow in Pohara Forest, Kendari is $D$. lanceolata or baoti (Tolakinese name).

D. lanceolata is one of seven spesies of Dryobalanops plants, and chemical and pharmacological aspects of $D$. lanceolata from Malaysia have been reported by Wibowo et al. [20]. This article reports isolation and structure determination of five stilbene oligomers from acetone extract of $D$. lanceolata stem barks from Kendari, Indonesia and their biological properties as antibacterial and anticancer. Three of five compounds from Kendari's Dryobalanops including balanocarpol, $\varepsilon$-viniferin, and $\alpha$-viniferin are the first time reported from the plant. In addition, biological activities toward bacteria E. coli ATCC 35219, S. aureus ATCC 25923 , and the cytotoxic property against human breast cancer cells T-47D have not been published yet.

\section{METHODS}

\section{General procedures}

Melting point (MP) of the isolated compounds was determined by "micro MP apparatus: Fisher John." The optical rotation established by polarimeter Perkin-Elmer 341 in $\mathrm{MeOH}$. Ultraviolet (UV) and Infrared (IR) spectra were measured with cary varian 100 concentration and Perkin-Elmer Spectrum One FT-IR spectrophotometer, respectively. spectra of ${ }^{1} \mathrm{H}$ and ${ }^{13} \mathrm{C}$ nuclear magnetic resonance (NMR) were determined by spectrophotometer JEOL LTD ECP 400, operated at $400 \mathrm{MHz}\left({ }^{1} \mathrm{H}\right)$ and $100.53 \mathrm{MHz}\left({ }^{13} \mathrm{C}\right)$, used acetone- $\mathrm{d}_{6}$ as solvent and TMS as internal standard. Separation and purification used thin-layer chromatography, vacuum liquid chromatography (VLC), and radial chromatography (RC). 
Sample

Stem barks of D. lanceolata got from Pohara Forest, Kendari, Sulawesi Tenggara. The plant was identified by staffs of Herbarium Bogoriense, Bogor, Indonesia.

\section{Extraction and isolation}

Powder of stem barks of D. lanceolata $(2.0 \mathrm{Kg})$ was extracted by acetone of $3 \times 5 \mathrm{~L}$ for $3 \times 24 \mathrm{hrs}$. The acetone extract was concentrated by rotary evaporator to get brown dark gum (116 g). All of acetone extract was fractionated by VLC using column with $\Phi 10 \mathrm{~cm}$, adsorben: Si-gel (150 g) and mixture of ethyl acetate: $\mathrm{N}$-hexane (30\%: $100-100 \%$ : $0 \%, \mathrm{MeOH}$ $100 \%)$ as eluent, produced 5 main fractions that are F1-F5 $(3.0,8.5,11.0$, 17.0 , and $19.0 \mathrm{~g})$. Separation of F2 (8.5 g) used VLC with column of $\Phi 10 \mathrm{~cm}$, gave 5 fractions F21-F25 $(0.4,1.2,1.8,1.1$, and 2.7 g). Purification of F22 using RC with eluent $10 \% \mathrm{MeOH}_{-} \mathrm{CHCl}_{3}$ yielded compound 1 (40 mg). Further separation of F24 employing the same procedure as F22 produced compound 2 (28 mg). Partition of F3 using VLC gave four fractions that are F31 (0.4 g), F32 (1.3 g), F33 (3.1 g), and F34 (3.1 g). Purification of F33 using VLC and RC yielded compound 3 (74 mg) and 4 (34 mg), respectively. Compound 5 (50 mg) came from separation and purification of F4 fraction which used the same method as purification of F33 fraction.

\section{Biological activities evaluation}

The antibacterial test was conducted by the agar dilution method using the general procedure outlined by Thakurta et al. [21]. The cultural concentration of bacteria was E. coli $=4.2 \times 10^{8} \mathrm{cfu} / \mathrm{mL}$ and $S$. Aureus = $3.2 \times 10^{7} \mathrm{cfu} / \mathrm{mL}$. The cytotoxic property toward human breast cancer cell lines T-47D was evaluated using MTT assays methods about $1 \times 10^{4}$ cells/well [22].

\section{RESULTS}

Data of spectroscopy and physical properties of isolated compounds

Compound 1, a yellow powder, MP $180-183^{\circ} \mathrm{C},[\alpha]_{\mathrm{D}}{ }^{20}-12^{\circ}(\mathrm{C} 0.1 \mathrm{MeOH})$. Spectra of UV (MeOH) $\lambda_{\text {maks }}(\log \varepsilon) 205$ (5.03), 220 (4.96), $284 \mathrm{~nm}$ (4.38), $(\mathrm{MeOH}+\mathrm{NaOH}) \lambda_{\text {maks }}(\log \varepsilon) 214$ (5.40), 247 (4.99), $295 \mathrm{~nm}$ (4.49). IR spectra $(\mathrm{KBr}) \hat{\mathrm{u}}_{\text {maks }}\left(\mathrm{cm}^{-1}\right) 3366(\mathrm{OH}), 1613,1512,1451$ (C=C aromatic), and 834 (para-disubstituted benzene). Spectra of ${ }^{1} \mathrm{H}$ NMR $\left(\mathrm{Me}_{2} \mathrm{CO}-\mathrm{d}_{6}\right.$, $400 \mathrm{MHz}$ ) and ${ }^{13} \mathrm{C} \mathrm{NMR}\left(\mathrm{Me}_{2} \mathrm{CO}-\mathrm{d}_{6}, 100 \mathrm{MHz}\right.$ ) (Table 1).

Compound 2, a yellow powder, MP $172-176^{\circ} \mathrm{C},[\alpha]_{\mathrm{D}}{ }^{20}-44^{\circ}(\mathrm{C} 0.1 \mathrm{MeOH})$. Spectra of UV (MeOH) $\lambda_{\text {maks }}(\log \varepsilon) 203$ (5.05), 230 (4.87), $324 \mathrm{~nm}$ (4.57), (MeOH+ NaOH) $\lambda_{\text {maks }}(\log \varepsilon) 211$ (5.52), $244(5.06), 347 \mathrm{~nm}$ (4.84). Spectra of IR (KBr) ن́v vaks $\left(\mathrm{cm}^{-1}\right) 3393(\mathrm{OH}), 1606,1513,1443$ ( $\mathrm{C}=\mathrm{C}$ aromatic), and 832 (para-disubstituted benzene). Spectra of ${ }^{1} \mathrm{H}$ NMR ( $\left.\mathrm{Me}_{2} \mathrm{CO}-\mathrm{d}_{6}, 400 \mathrm{MHz}\right) \delta_{\mathrm{H}}(\mathrm{ppm}) 7.14(2 \mathrm{H}, \mathrm{d}, \mathrm{J}=8.4 \mathrm{~Hz}, \mathrm{H}-2 / 6 \mathrm{a})$, $6.77(2 \mathrm{H}, \mathrm{d}, \mathrm{J}=8.4 \mathrm{~Hz}, \mathrm{H}-3 / 5 \mathrm{a}), 5.38(1 \mathrm{H}, \mathrm{d}, \mathrm{J}=6.6 \mathrm{~Hz}, \mathrm{H}-7 \mathrm{a}), 4.35$ (1H, d,

Table 1: Biological activities of the isolated compounds

\begin{tabular}{|c|c|c|c|}
\hline \multirow[t]{3}{*}{ Compound(s) } & \multicolumn{3}{|c|}{ Biological activities (mean \pm SD*) } \\
\hline & \multicolumn{2}{|c|}{$\begin{array}{l}\text { Diameter of } \\
\text { inhibition zone }(\mathrm{mm})\end{array}$} & \multirow{2}{*}{$\begin{array}{l}\mathrm{IC}_{50}(\mu \mathrm{M}) \\
\mathrm{T}-47 \mathrm{D}\end{array}$} \\
\hline & E. coli & S. aureus & \\
\hline Balanocarpol & $9 \pm 0.17$ & $13 \pm 0.12$ & $98.17 \pm 0.41$ \\
\hline$\varepsilon$-Viniferin & $11 \pm 0.22$ & $7 \pm 0.17$ & $34.13 \pm 0.15$ \\
\hline$\alpha$-Viniferin & $8 \pm 0.20$ & $8 \pm 0.16$ & $84.79 \pm 0.24$ \\
\hline Vaticanol B & $5 \pm 0.12$ & $4 \pm 0.14$ & $119.30 \pm 0.54$ \\
\hline Hopeaphenol & $6 \pm 0.16$ & $8 \pm 0.11$ & $52.04 \pm 0.26$ \\
\hline $\begin{array}{l}\text { Tetracycline (antibacterial } \\
\text { standard) }\end{array}$ & $14 \pm 0.14$ & $19 \pm 0.12$ & - \\
\hline $\begin{array}{l}\text { Doxorubicine (anticancer } \\
\text { standard) }\end{array}$ & - & - & $2.35 \pm 0.08$ \\
\hline
\end{tabular}

*SD: Standard deviation, triplicates; diameter of Whatman

paper $=6 \mathrm{~mm}$, $($ Balanocarpol $)=(\varepsilon$-viniferin $)=(\alpha$-viniferin $)=($ vaticanol B $)$

$=($ hopeaphenol $)=10000 \mu \mathrm{g} / \mathrm{mL}$; control (teracyclin $30 \mu \mathrm{g} / \mathrm{disc})$. For

MTT assays, (sample) $=100 \mathrm{mg} / \mathrm{mL}, 50 \mathrm{mg} / \mathrm{mL}, 25 \mathrm{mg} / \mathrm{mL}, 12.5 \mathrm{mg} / \mathrm{mL}$,

$6.25 \mathrm{mg} / \mathrm{mL}$, and $3.125 \mathrm{mg} / \mathrm{mL}$
$\mathrm{J}=6.6 \mathrm{~Hz}, \mathrm{H}-8 \mathrm{a}), 6.23(2 \mathrm{H}, \mathrm{d}, \mathrm{J}=2.2 \mathrm{~Hz}, \mathrm{H}-10 / 14 \mathrm{a}), 6.20$ (1H, br d, H-12a), $7.15(2 \mathrm{H}, \mathrm{d}, \mathrm{J}=8.4 \mathrm{~Hz}, \mathrm{H}-2 / 6 \mathrm{~b}), 6.65$ (2H, d, J=8.4 Hz, H-3/5b), $6.83(1 \mathrm{H}$, d, J=16.3 Hz, H-7b), 6.58 (1H, d, J=16.3 Hz, H-8b), 6.26 (1H, d, J=2.1 Hz, $\mathrm{H}-12 \mathrm{~b})$, and $6.64(1 \mathrm{H}, \mathrm{d}, \mathrm{J}=2.1 \mathrm{~Hz}, \mathrm{H}-14 \mathrm{~b})$.

Compound 3, a pale brown powder, MP $222-224^{\circ} \mathrm{C}$ and $[\alpha]_{D}{ }^{20}+60^{\circ}$ (C $0.1 \mathrm{MeOH})$. Spectra of UV (MeOH) $\lambda_{\text {mats }}$ (log $\varepsilon$ ) 229 (4.57) and $286 \mathrm{~nm}$ (4.19). Spectra of UV $(\mathrm{MeOH}+\mathrm{NaOH}) \lambda_{\text {maks }}(\log \varepsilon) 251$ (4.78), $295 \mathrm{~nm}$ (4.32), Spectra of IR (KBr) $\mathrm{u}_{\text {maks }} 3307 \mathrm{~cm}^{-1}(-\mathrm{OH}), 1614,1515$, and $1486 \mathrm{~cm}^{-1}$ ( $\mathrm{C}=\mathrm{C}$ benzene), and $831 \mathrm{~cm}^{-1}$ (para-disubstituted benzene). Spectra of ${ }^{1} \mathrm{H}$ NMR (aseton- $\left.\mathrm{d}_{6}, 400 \mathrm{MHz}\right) \delta$ ppm: 7,26 $(2 \mathrm{H}, \mathrm{d}$, $\mathrm{J}=8.5 \mathrm{~Hz}, \mathrm{H}-2 / 6 \mathrm{~b}), 7.08(2 \mathrm{H}, \mathrm{d}, \mathrm{J}=8.5 \mathrm{~Hz}, \mathrm{H}-2 / 6 \mathrm{c}), 7.06(2 \mathrm{H}, \mathrm{d}, \mathrm{J}=8.5 \mathrm{~Hz}$, $\mathrm{H}-2 / 6 \mathrm{a}), 6.82(2 \mathrm{H}, \mathrm{d}, \mathrm{J}=8.5 \mathrm{~Hz}, \mathrm{H}-3 / 5 \mathrm{c}), 6.80$ (2H, d, J=8.5 Hz, H-3/5b), 6.76 (1H, d, J=1.5 Hz, H-11b), 6.75 (2H, d, J=8.5 Hz, H-3/5a), $6.63(1 \mathrm{H}, \mathrm{d}, \mathrm{J}=2.0 \mathrm{~Hz}, \mathrm{H}-11 \mathrm{c}), 6.27(1 \mathrm{H}, \mathrm{d}, \mathrm{J}=1.5 \mathrm{~Hz}, \mathrm{H}-13 \mathrm{~b}), 6.25(1 \mathrm{H}$, d, J=2.0 Hz, H-13a), 6.25 (1H, d, J= 2,0 Hz, H-13c), 6,10 (1H, br s, H-7a), 6,01 (1H, d, J=2,0 Hz, H-11a), 5,96 (1H, d, J=9.5 Hz, H-7b), $4.93(1 \mathrm{H}$, d, J=6.5 Hz, H-7c), $4.73(1 \mathrm{H}, \mathrm{d}, \mathrm{J}=9.5 \mathrm{~Hz}, \mathrm{H}-8 \mathrm{~b}), 4.65(1 \mathrm{H}, \mathrm{d}, \mathrm{J}=6.5 \mathrm{~Hz}$, $\mathrm{H}-8 \mathrm{c}), 3.99$ (1H, br s, H-8a), and $\mathrm{OH}(8.61 ; 8.57$; 8.51; 8.43; 8.42; and 8.41 each s, $1 \mathrm{H}$ ). Spectra of ${ }^{13} \mathrm{C}$ NMR (aseton-d, $100 \mathrm{MHz}, \mathrm{APT}$ ) $\delta$ ppm: 161.6 (C-14a), 161.1 (C-14c), 160.8 (C-12c), 160.6 (C-14b), 159.3 (C-12a), 159.34 (C-12b), 158.3 (C-4c), 158.2 (C-4b), 157.8 (C-4a), 141.2 (C-10a), 138.7 (C-10c), 139.7 (C-10b), 132.5 (C-1c), 132.2 (C-1b), 132.0 (C-1a), 128.6 (C-2/6c), 128.15 (C-2/6b), 128.1 (C-2/6a), 120.9 (C-9b), 119.0 (C-9c), 118.8 (C-9a), 116.1 (C-3/5c), 116.1 (C-3/5 b), 115.7 (C-3/5a), 108.5 (C-11a), 106.2 (C-11b), 105.8 (C-11c), 98.0 (C-13a), 96.9 (C-13c), 96.6 (C-13b), 95.6 (C-7c), 90.0 (C-7b), 86.4 (C-7a), 55.6 (C-8c), 52.8 (C-8b), and 46.4 (C-8a).

Compound 4, a pale brown powder, MP $207-210^{\circ} \mathrm{C},[\alpha]_{\mathrm{D}}{ }^{20}-35^{\circ}(\mathrm{C} 0.1$ $\mathrm{MeOH}$ ); UV (MeOH) $\lambda_{\text {maks }}(\log \varepsilon) 203$ (5.09), 229 (4.89), $284 \mathrm{~nm}$ (4.19), absorption of UV (MeOH+NaOH) $\lambda_{\text {maks }}(\log \varepsilon) 207$ (5.36), 247 (4.59), $286 \mathrm{~nm}$ (4.19), Spectra of IR (KBr) $\mathrm{u}_{\text {maks }}\left(\mathrm{cm}^{-1}\right) 3370(\mathrm{OH}), 1614,1514$, and 1454 (benzene), and 832 (para-disubstituted benzene). Spectra of ${ }^{1} \mathrm{H}$ NMR (aseton-d, $\left.400 \mathrm{MHz}\right) \delta$ ppm: 7.22 (2H, d, J=8.4 Hz, H-2/6a), 6,76 (2H, d, J=8.4 Hz, H-3/5a), 5,75 (1H, d, J=11.2 Hz, H-7a), $4.41(1 \mathrm{H}$, d, J=11.2 Hz, H-8a), $6.26(1 \mathrm{H}, \mathrm{d}, \mathrm{J}=2.2 \mathrm{~Hz}, \mathrm{H}-12 \mathrm{a}), 6.10(1 \mathrm{H}, \mathrm{d}, \mathrm{J}=2.2 \mathrm{~Hz}$, $\mathrm{H}-14 \mathrm{a}), 7.14$ (2H, d, J=8.4 Hz, H-2/6b), 6.67 (2H, d, J=8.4 Hz, H-3/5b), 5.19 (1H, d, J=3.7 Hz, H-7b), 3.09 (1H, br d, H-8b), 6.03 (1H, br s, H-12b), $6.38(2 \mathrm{H}, \mathrm{d}, \mathrm{J}=8.4 \mathrm{~Hz}, \mathrm{H}-2 / 6 \mathrm{c}), 6.48(2 \mathrm{H}, \mathrm{d}, \mathrm{J}=8.4 \mathrm{~Hz}, \mathrm{H}-3 / 5 \mathrm{c}), 4.08(1 \mathrm{H}, \mathrm{t}$, $\mathrm{J}=10.5 ; 10.0 \mathrm{~Hz}, \mathrm{H}-7 \mathrm{c}), 4.52$ (1H, d, J=10.2 Hz, H-8c), 6.17 (1H, d, J=2.2 Hz, $\mathrm{H}-12 \mathrm{c}), 6.45(1 \mathrm{H}, \mathrm{d}, \mathrm{J}=2.2 \mathrm{~Hz}, \mathrm{H}-14 \mathrm{c}), 7.17(2 \mathrm{H}, \mathrm{d}, \mathrm{J}=8.4 \mathrm{~Hz}, \mathrm{H}-2 / 6 \mathrm{~d})$, $6.75(2 \mathrm{H}, \mathrm{d}, \mathrm{J}=8.4 \mathrm{~Hz}, \mathrm{H}-3 / 5 \mathrm{~d}), 5.35(1 \mathrm{H}, \mathrm{d}, \mathrm{J}=5.1 \mathrm{~Hz}, \mathrm{H}-7 \mathrm{~d}), 4.66(1 \mathrm{H}$, d, J=5.1Hz, H-8d), $6.08(2 \mathrm{H}$, br s, H-10/14d), $6.26(1 \mathrm{H}, \mathrm{t}, \mathrm{J}=2.2 \mathrm{~Hz}$, $\mathrm{H}-12 \mathrm{~d}$ ), Spectra of ${ }^{13} \mathrm{C}$ NMR (aseton- $\left.{ }_{6}, 100 \mathrm{MHz}, \mathrm{APT}\right) \delta \mathrm{ppm:} 161.8$ (C-11c), 159.3 (C-11d), 159.5 (C-13c), 159.3 (C-13d), 158.9 (C-11b), 158.7 (C-4a), 158.1 (C-4d), 156.8 (C-13a), 156.4 (C-4c), 156.0 (C-4b), 155.8 (C-11a), 155.0 (C-13b), 148.1 (C-9d), 143.2 (C-9b), 141.9 (C-9a), 141.8 (C-9c), 134.7 (C-1d), 133.6 (C-1b), 131.5 (C-1c), 130.9 (C-1a), 130.8 (C-2/6b), 130.3 (C-2/6a), 129.3 (C-2/6c), 128.3 (C-2/6d), 124.6 (C-10a), 123.4 (C-10c), 122.3 (C-14b), 116.1 (C-3/5d), 116.08 (C-3/5a), 115.9 (C-3/5c), 115.8 (C-10b), 115.5 (C-3/5b), 107.6 (C-10/14d), 107.1 (C-14c), 105.8 (C-14a), 102.3 (C-12d), 101.7 (C-12a), 96.6 (C-12b), 95.8 (C-12c), 94.7 (C-7d), 90.5 (C-7a), 57.7 (C-8d), 57.6 (C-7c), 53.3 (C-8b), 49.3 (C-8c), 48.9 (C-8a), and 37.2 (C-7b).

Compound 5, a white powder, MP $160-164^{\circ} \mathrm{C},[\alpha]_{\mathrm{D}}{ }^{20}+138^{\circ}$ (C 0.1 $\mathrm{MeOH}) ; \mathrm{UV}(\mathrm{MeOH}) \lambda_{\text {maks }}(\log \varepsilon) 203$ (5.10), 230 (4.88), and $283 \mathrm{~nm}$ $(4,23)$. Absorption of UV (MeOH+NaOH) $\lambda_{\text {maks }}(\log \varepsilon) 207$ (5.27), 250 (4.51), $288 \mathrm{~nm}$ (3.94). Spectra of IR (KBr) $\mathrm{u}_{\text {maks }}\left(\mathrm{cm}^{-1}\right) 3419(\mathrm{OH})$, 2927 ( $\mathrm{CH}$ aliphatic), 1614, 1512, and 1455 (benzene), and 834 (paradisubstituted benzene). Spectra of ${ }^{1} \mathrm{H}$ NMR (aseton- $\left.\mathrm{d}_{6}, 400 \mathrm{MHz}\right) \delta$ ppm: 7.52 (2H, d, J=8.8, H-2/6a), 6.98 (2H, d, J=8.8, H-3/5a), $5.63(1 \mathrm{H}$, d, J=10.3, H-7a), 5.42 (1H, d, J=10.3, H-8a), 6.36 (1H, d, J=2.2, H-12a), $6.28(1 \mathrm{H}, \mathrm{d}, \mathrm{J}=2.2, \mathrm{H}-14 \mathrm{a}), 6.92(2 \mathrm{H}, \mathrm{d}, \mathrm{J}=8.4, \mathrm{H}-2 / 6 \mathrm{~b}), 6.58(2 \mathrm{H}, \mathrm{d}, \mathrm{J}=8.4$, $\mathrm{H}-3 / 5 \mathrm{~b}), 5.81$ (1H, br s, H-7b), $3.96(1 \mathrm{H}, \mathrm{s}, \mathrm{H}-8 \mathrm{~b}), 5.75(1 \mathrm{H}, \mathrm{d}, \mathrm{J}=2.1$, $\mathrm{H}-12 \mathrm{~b})$, and $5.18(1 \mathrm{H}, \mathrm{d}, \mathrm{J}=2.1, \mathrm{H}-14 \mathrm{~b})$. Spectra of ${ }^{13} \mathrm{C}$ NMR (aseton-d, $100 \mathrm{MHz}, \mathrm{APT}) \delta$ ppm: 133.8 (C-1a), 131.1 (C-2/6a), 116.9 (C-3/5a), 159.1 (C-4a), 94.6 (C-7a), 54.2 (C-8a), 141.1 (C-9a), 118.8 (C-10a), 
160.6 (C-11a), 102.7 (C-12a), 158.2 (C-13a), 107.4 (C-14a), 138.2 (C1b), 130.1 (C-2/6b), 114.9 (C-3/5b), 155.1 (C-4b), 44.1 (C-7b), 53.4 (C-8b), 142.2 (C-9b), 117.7 (C-10b), 159.1 (C-11b), 95.3 (C-12b), 156.9 (C-13b), and $110.2(\mathrm{C}-14 \mathrm{~b})$.

\section{Biological activities data}

The biological activities data of isolated compounds against bacteria and T-47D cancer cell lines are displayed in Table 1.

\section{DISCUSSION}

Stilbene monomer (resveratrol) comprises 14 carbon atoms and structure pattern $\mathrm{C}_{6}-\mathrm{C}_{2}-\mathrm{C}_{6}$. The research has isolated and identified five stilbene oligomers from acetone extract of $D$. lanceolata stem barks. A total of 2 compounds have 28 carbon atoms or stilbene dimmers, a compound has 42 carbon atoms or stilbenes trimer, and two compounds have 56 carbon atoms or stilbene tetramer. The compound structures are displayed in the Fig. 1.

All isolated compounds are known compounds so the structures are determined by comparing the spectroscopic data of isolated compounds with similar data from references. For example, isolate 1, the spectrum data of ${ }^{1} \mathrm{H}$ NMR and ${ }^{13} \mathrm{C}$ NMR has a high similarity parameter with balanocarpol $\left(1^{*}\right)$ [23]. It can be concluded that compound 1 is balanocarpol as displayed in Table 2 .

In the same way as structure determination of balanocarpol, the compounds 2, 3, 4, and 5 are $\varepsilon$-viniferin [24], $\alpha$-viniferin [25], vaticanol B [26], and hopeaphenol [27], respectively. Refers to a previous study [20], three compounds were isolated from $D$. lanceolata from Kendari, namely, balanocarpol, $\varepsilon$-viniferin, and $\alpha$-viniferin, not reported from Malaysia's $D$. lanceolata. These data complemented the diversity of $D$. lanceolata's stilbene oligomers. Two compounds, vaticanol $\mathrm{B}$ and hopeaphenol, have been reported previously that thought to be characteristic of D. lanceolata.

According to biological activity data in Table 1, biological activity against bacteria indicated that $\varepsilon$-viniferin is the most active compounds toward E. coli, which followed by balanocarpol, $\alpha$-viniferin, hopeaphenol, and vaticanol B. While the activity against $S$. aureus, balanocarpol is the most active compound followed by $\alpha$-viniferin, hopeaphenol, $\varepsilon$-viniferin, and vaticanol B. $\varepsilon$-Viniferin and balanocarpol are stilbene dimers, consist of two unit stilbenes, means having a smaller molecular size than the others. It is estimated that activity of stilbene derivative against bacteria is influenced by the size of the molecule which can affect molecular penetration [28].

In general, stilbene derivatives have cytotoxic potency toward cancer cell lines not only from Dipterocarpaceae but also from Gnetaceae, for example, gnetin $\mathrm{C}$ and gnemonoside A, two stilbene dimers from Gnetum gnemon [29] or other phenolic compounds such as curcumin [30]. The cytotoxic properties against human breast cancer cell lines T-47D showed that all isolated compounds are less active than standard (doxorubicin). For isolated compounds, $\varepsilon$-viniferin is the most active compound, followed by hopeaphenol, $\alpha$-viniferin, balanocarpol, and vaticanol B. $\varepsilon$-Viniferin has intact stilbene unit and all carbon atoms have orbital hybrid sp2. Consequently, $\varepsilon$-viniferin becomes richer phi electrons with the equitable electrons distribution from the ring B1 to B2 through electron delocalization. It will produce a more stable radical that can inhibit cancer cell growth [31]. Biological activity of hopeaphenol and $\alpha$-viniferin against human breast cancer cell lines T-47D thought to be caused by the density of the compound [32]. Both of these compounds have a symmetrical plane in their structure. This causes the density of molecules into larger, like hopeaphenol, this compound has a small volume identical with stilbene dimer, but its molecular weight as a tetramer. Hopeaphenol as a tetramer has a molecular weight greater than two times the dimer, because there is a plane of symmetry in the structure hopeaphenol, the volume of hopeaphenol structure similar to the dimer. Consequently, hopeaphenol density becomes greater and more active compound against cancer cell lines. The sequence of cytotoxic properties of stilbene oligomers toward T-47D cell lines was identic to the sequence of the cytotoxic properties of the compounds against murine leukemia P-388 cells that are hopeaphenol $(5.7 \pm 0.3 \mathrm{mM}), \alpha$-viniferin $(25.8 \pm 0.7 \mu \mathrm{M})$, balanocarpol $(33.6 \pm 8.3 \mu \mathrm{M})$, and vaticanol B $(56.8 \pm 2.3 \mu \mathrm{M})[1]$.

\section{CONCLUSION}

Five stilbene oligomers have been isolated and identified from the stem barks of $D$. lanceolata, namely, balanocarpol (1), $\varepsilon$-viniferin (2) (stilbene

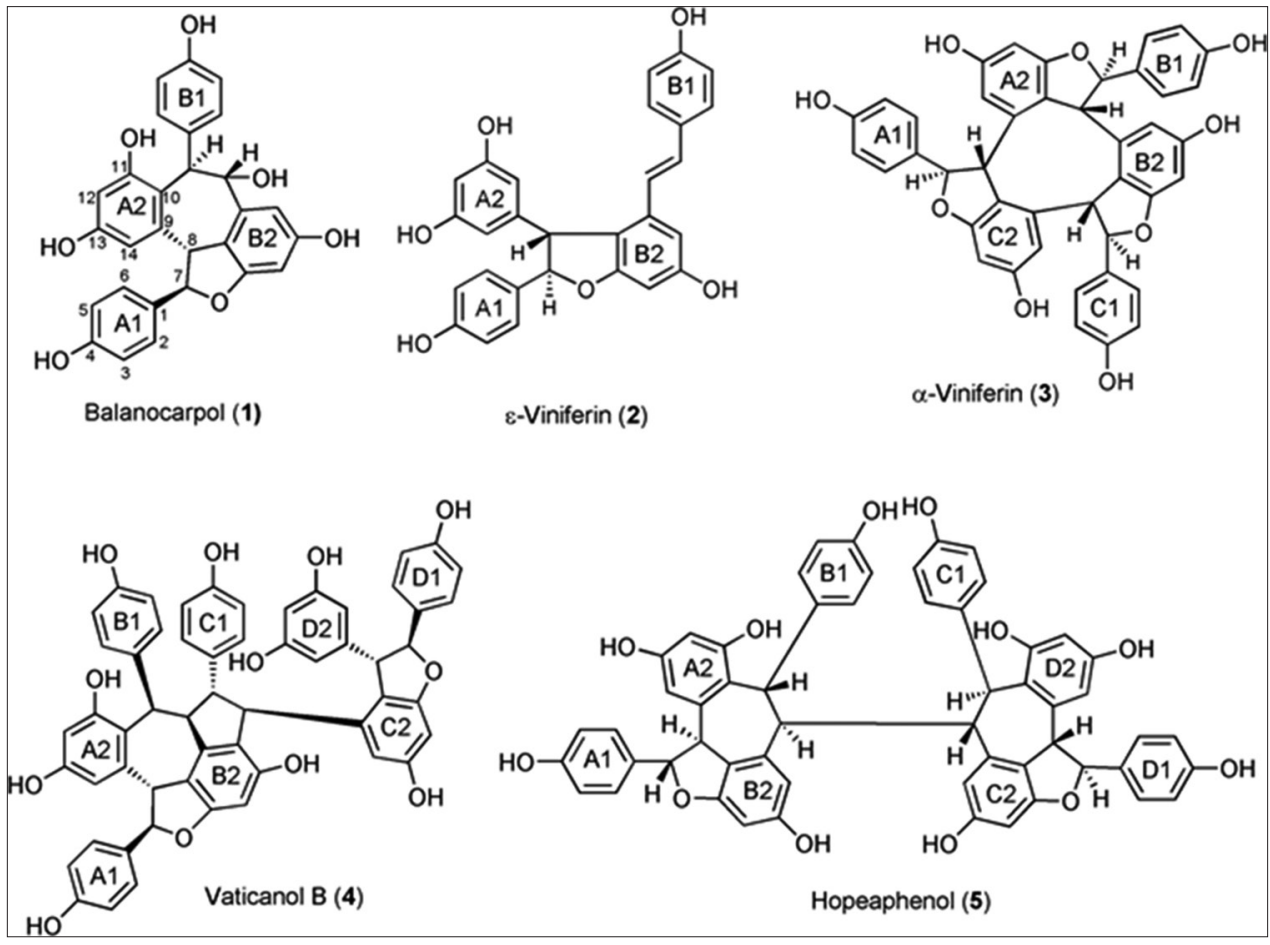

Fig. 1: Compound structures from Dryobalanops lanceolata stem barks (1 and 2) stilbene dimers, (3) stilbene trimer, and (4 and 5) stilbene tetramers 
Table 2: Spectra of ${ }^{1} \mathrm{H}$ NMR of balanocarpol (1)

\begin{tabular}{|c|c|c|c|c|}
\hline \multirow[t]{2}{*}{ Number of component } & \multicolumn{2}{|l|}{$\delta_{\mathrm{H}}$ (mult., J in Hz) } & \multicolumn{2}{|l|}{$\delta_{c}$} \\
\hline & 1 & $1^{*}$ & 1 & $1^{*}$ \\
\hline $1 \mathrm{a}$ & - & - & 133.2 & 133.5 \\
\hline $2(6) \mathrm{a}$ & $7.48(2 \mathrm{H}, \mathrm{d}, 8.4)$ & $7.50(2 \mathrm{H}, \mathrm{d}, 8.3)$ & 131.3 & 131.5 \\
\hline $3(5) \mathrm{a}$ & $6.94(2 \mathrm{H}$, d. 8.4$)$ & $6.95(2 \mathrm{H}, \mathrm{d}, 8.3)$ & 113.9 & 114.2 \\
\hline $4 a$ & - & - & 155.5 & 155.8 \\
\hline $7 \mathrm{a}$ & $5.69(1 \mathrm{H}, \mathrm{d}, 9.5)$ & $5.69(1 \mathrm{H}, \mathrm{d}, 9.3)$ & 72.9 & 73.2 \\
\hline $8 a$ & $5.15(1 \mathrm{H}, \mathrm{d}, 9.5)$ & $5.16(1 \mathrm{H}, \mathrm{brd}, 9.3)$ & 50.0 & 50.3 \\
\hline $9 \mathrm{a}$ & - & - & 140.6 & 140.8 \\
\hline $10 \mathrm{a}$ & - & - & 113.6 & 113.8 \\
\hline $11 \mathrm{a}$ & - & - & 159.5 & 159.7 \\
\hline $12 \mathrm{a}$ & $6.09(1 \mathrm{H}, \mathrm{d}, 2.2)$ & $6.09(1 \mathrm{H}, \mathrm{br} \mathrm{s})$ & 94.8 & 95.1 \\
\hline $13 a$ & - & - & 158.9 & 159.2 \\
\hline $14 \mathrm{a}$ & $5.95(1 \mathrm{H}, \mathrm{d}, 2.2)$ & $5.96(1 \mathrm{H}, \mathrm{d}, 2.3)$ & 104.2 & 104.4 \\
\hline $1 b$ & - & - & 133.4 & 133.7 \\
\hline $2(6) b$ & $6.73(2 \mathrm{H}, \mathrm{d}, 8.4)$ & $6.75(2 \mathrm{H}, \mathrm{d}, 8.3)$ & 130.3 & 130.5 \\
\hline $3(5) \mathrm{b}$ & $6.41(2 \mathrm{H}, \mathrm{d}, 8.4)$ & $6.42(2 \mathrm{H}, \mathrm{d}, 8.3)$ & 116.2 & 116.4 \\
\hline $4 \mathrm{~b}$ & - & - & 158.3 & 158.6 \\
\hline $7 b$ & $4.89(1 \mathrm{H}, \mathrm{br} \mathrm{s})$ & $4.90(1 \mathrm{H}, \mathrm{br} \mathrm{s})$ & 52.1 & 52.3 \\
\hline $8 b$ & $5.38(1 \mathrm{H}, \mathrm{br} \mathrm{s})$ & $5.40(1 \mathrm{H}, \mathrm{br} \mathrm{s})$ & 93.3 & 93.5 \\
\hline $9 \mathrm{~b}$ & - & - & 142.6 & 142.8 \\
\hline $10 \mathrm{~b}$ & - & - & 120.2 & 120.4 \\
\hline $11 \mathrm{~b}$ & - & - & 157.2 & 157.4 \\
\hline $12 \mathrm{~b}$ & $6.18(1 \mathrm{H}, \mathrm{d}, 2.2)$ & $6.20(1 \mathrm{H}, \mathrm{br} \mathrm{s})$ & 101.8 & 102.0 \\
\hline $13 b$ & - & - & 156.5 & 156.9 \\
\hline $14 \mathrm{~b}$ & $6.24(1 \mathrm{H}, \mathrm{d} .2 .2)$ & $6.26(1 \mathrm{H}, \mathrm{d}, 2.0)$ & 106.5 & 106.8 \\
\hline \multirow[t]{6}{*}{$\mathrm{OH}$} & $4.41(\mathrm{br} \mathrm{d}, 4.4)$ & $4.36(\mathrm{~d}, 4.4, \mathrm{C}-8 \mathrm{~b})$ & & \\
\hline & $8.65 ; 8.09 ; 8.06 ; 7.91 ; 7.81$ (br s) & 7.74 (br s, C-4a) & & \\
\hline & & 7.85 (br s, C-13b) & & \\
\hline & & 7.79 (br s, C-11a) & & \\
\hline & & 8.04 (br s, C-13a) & & \\
\hline & & 8.56 (br s, C-4b) & & \\
\hline
\end{tabular}

Measured in acetone- $\mathrm{d}_{6}\left({ }^{1} \mathrm{H}, 400 \mathrm{MHz} ;{ }^{13} \mathrm{C}\right.$ NMR $\left.100 \mathrm{MHz}\right) *$ [23], NMR: Nuclear magnetic resonance

dimer), and $\alpha$-viniferin (3) (stilbene trimer) and two stilbene tetramers that are vaticanol B (4) and hopeaphenol (5). $\varepsilon$-Viniferin was the most active compound against E. coli and balanocarpol was the most active substance toward $S$. aureus. Cytotoxic properties against human breast cancer cell lines T-47D indicated that $\varepsilon$-viniferin is the most active compound.

\section{ACKNOWLEDGMENTS}

We would like to thank the Ministry of Research, Technology and Higher Education of the Republic of Indonesia for the research grants "Hibah Fundamental 2009 and Hibah Kompetensi 2015."

\section{REFERENCES}

1. Sahidin, Hakim EH, Juliawaty LD, Syah YM, Din LB, Ghisalberti EL, et al. Cytotoxic properties of oligostilbenoids from the tree barks of Hopea dryobalanoides. Z Naturforsch C 2005;60(9-10):723-7.

2. Juliawaty LD, Sahidin, Hakim EH, Achmad SA, Syah YM, Latip J, et al. A 2-arylbenzofuran derivative from Hopea mengarawan. Nat Prod Commun 2009;4(7):947-50.

3. Muhammad N, Din LB, Sahidin I, Hashim SF, Ibrahim N, Zakaria Z, et al. Acuminatol and other antioxidative resveratrol oligomers from the stem bark of Shorea acuminata. Molecules 2012;17(8):9043-55.

4. Sahidin I, Ardiansyah S, Taher M, Manggau MA. Terpenoids from the stem bark of Jatropha plants and their biological activities. Makara Sains 2011;15(2):106-10

5. Sahidin I, Yamin N, Ginting S, Manggau MA, Lukman. Cytotoxic potency of diterpenes from Jatropha plants. Int J Pharm Pharm Sci 2013;5(3):3-6.

6. Sabandar CW, Ahmat N, Jaafar FM, Sahidin I. Medicinal property. phytochemistry and pharmacology of several Jatropha species (Euphorbiaceae): A review. Phytochemistry 2013;85:7-29.

7. Rosandy AR, Din LB, Yaacob WA, Yusoff NI, Sahidin I, Latip J, et al. Isolation and characterization of compounds from the stem bark of Uvaria rufa (Annonaceae). MJAS 2013;17(1):50-8.

8. Al Muqarrabun LM, Ahmat N, Ruzaina SA, Ismail NH, Sahidin I.
Medicinal uses. Phytochemistry and pharmacology of Pongamia pinnata (L.) Pierre: A review. J Ethnopharmacol 2013;150(2):395-420.

9. Ruslin M, Asmawi MZ, Rianse U, Sahidin I, Dhianawaty D, Soemardji AA, et al. Anti-hypertensive activity of Alang-alang (Imperata cylindrica L.) Beauv. root methanolic extract on male Wistar rat. Int J Res Pharm Sci 2013;4(4):537-42.

10. Sahidin I, Nohong N, Sani A, Manggau MA, Sukohar A, Widodo H, et al. Radical scavenging activity of triterpene steroids from stem of Polygonum pulchrum B1. Int J Pharm Pharm Sci 2014;6(8):350-4.

11. Sahidin I, Suwandi A, Nohong N, Manggau MA. Profile of anticancer and radical scavenging activities of steroids from stems of Polygonum pulchrum. IJPSR 2015;6(5):2178-84.

12. Jalil J, Sabandar CW, Ahmat N, Jamal JA, Jantan I, Aladdin NA, et al. Inhibitory effect of triterpenoids from Dillenia serrata (Dilleniaceae) on prostaglandin E2 production and quantitative HPLC analysis of its koetjapic acid and betulinic acid contents. Molecules 2015;20(2):3206-20.

13. Ashton PS. Flora Malesiana Spermatophyta I. The Hague: Martinus Nijhoff; 1983. p. 391-436.

14. Aslam MS, Ahmad MS, Mamat AS. A phytochemical, ethnomedicinal and pharmacological review of genus Dipterocarpus. Int J Pharm Pharm Sci 2015;7(4):27-38.

15. Shettar AK, Vedhamurthy AB. Studies on in vitro antidiabetic activities of Hopea longa and Vitex leucoxylon. Int J Pharm Pharm Sci 2017;9(2):263-7.

16. Wibowo A, Ahmat N, Hamzah AS, Sulfian AS, Ismail NH, Ahmad R, et al. Malaysianol A, a new trimer resveratrol oligomer from the stem bark of Dryobalanops aromatica. Fitoterapia 2011;82:676-81.

17. Wibowo A, Ahmat N, Hamzah AS, Ismail NH, Ahmad R, Jaafar FM. Resveratrol oligomers from the stem bark of Dryobalanops aromatica. Biochem Syst Ecol 2012;40:62-4.

18. Wibowo A, Ahmat N, Hamzah AS, Latif FA, Norrizah JS, Khong HY, et al. Identification and biological activity of secondary metabolites from Dryobalanops beccarii. Phytochem Lett 2014;9:117-22.

19. Syah YM, Aminah NS, Hakim EH, Aimi N, Kitajima M, Takayama J, et al. Two oligostilbenes, cis- and trans-diptoindonesin B from Dryobalanops oblongifolia. Phytochemistry 2003;63:913-7. 
20. Wibowo A, Ahmat N, Hamzah AS, Low AL, Mohamad SA, Khong HY, et al. Malaysianol B, an oligostilbenoid derivative from Dryobalanops lanceolata. Fitoterapia 2012;83:1569-75.

21. Thakurta P, Bhowmik P, Mukherjee S, Hajra TK, Patra A, Bag PK. Antibacterial, antisecretory and antihemorrhagic activity of Azadirachta indica used to treat cholera and diarrhea in India. J Ethnopharmacol 2007;111:607.

22. Soundararajan R, Prabha P, Rai U, Dixit A. Antileukemic potential of Momordica charantia seed extracts on human myeloid leukemic HL60 cells, evidence-based. Complement Altern Med 2012;10:1-10.

23. Tanaka T, Ito T, Ido Y, Nakaya K, Iinuma M, Chelladurai V. Hopeafuran and a C-glucosyl resveratrol isolated from stem wood of Hopea utilis. Chem Pharm Bull 2001;49(6):785-7.

24. Oshima Y, Ueno Y. Ampelopsins D, E, H, and cis-ampelopsin E, oligostilbenes from Ampelopsin brevipedunculata var. Hancei roots. Phytochemistry 1993;33(1):179-82.

25. Kitanaka S, Ikezawa T, Yosukawa K, Yamanouchi S, Takido M, Sung HK, et al. $(+)-\alpha$-viniferin, an anti-inflammatory compound from Caragana chamlagu Lamarck. Chem Pharm Bull 1990;38(2):432-5
26. Tanaka T, Ito T, Ido Y, Son TK, Nakaya K, Iinuma M, et al. Stilbenoids in stem bark of Hopea parviflora. Phytochemistry 2000;53:1009-14.

27. Ito J, Niwa M, Oshima Y. A new hydroxystilbene tetramer named isohopeaphenol from Vitis vinifera. Heterocycles 1997;45(9):1809-13.

28. Ebejer JP, Charlton MH, Finn PW. Are the physicochemical properties of antibacterial compounds really different from other drugs? J Cheminform 2016;8:30.

29. Mun'im A, Munadhil MA, Puspitasari N, Azminah, Yanuar A. Angiotensin converting enzyme inhibitory activity of Melinjo (G. gnemon) seed extracts and molecular docking of its stilbene constituents. Asian J Pharm Clin Res 2017;10(3):243-8.

30. Steffi PF, Srinavasan M. Curcumin, a potent anticarcinogenic polyphenol-a review. Asian J Pharm Clin Res 2014;7(Suppl 2):1-8.

31. Khaledi H, Alhadi AA, Yehye WA, Ali HM, Abdulla MA, Hassandravish P. Antioxidant, cytotoxic activities, and structureactivity relationship of gallic acid-based indole derivatives. Arch Pharm Chem Life Sci 2011;344:703-9.

32. Chu C, Xu P, Zhao H, Chen Q, Chen D, Hu H, et al. Effect of surface ligand density on cytotoxicity and pharmacokinetic profile of docetaxel loaded liposome. Asian J Pharm Sci 2016;11:665-71. 